\title{
Primary intrahepatic cholangiocarcinoma with sarcomatous stroma: case report and review of the literature
}

Kyohei Yugawa ${ }^{1,2^{*}} \mathbb{D}$, Tomoharu Yoshizumi ${ }^{1}$, Yohei Mano ${ }^{1}$, Noboru Harada', Shinji Itoh ${ }^{1}$, Toru Ikegami ${ }^{1}$, Yuji Soejima', Nobuhiro Fujita ${ }^{3}$, Kenichi Kohashi ${ }^{2}$, Shinichi Aishima ${ }^{4}$, Yoshinao Oda ${ }^{2}$ and Masaki Mori ${ }^{1}$

\begin{abstract}
Background: Hepatic carcinosarcomas, which include both carcinomatous and sarcomatous elements, are uncommon in adults. Although carcinosarcoma in hepatocellular carcinoma is occasionally reported, carcinosarcoma in intrahepatic cholangiocarcinoma (ICC) is an extremely rare ICC variant. Few such cases have been reported in English and no large study of its clinicopathological features exists.

Case presentation: Here, we report a 60-year-old man with an asymptomatic hepatic B infection who developed hepatic carcinosarcoma from an otherwise normal liver. The 6.0-cm tumor was accidentally discovered by PET-CT in a cancer examination. Serum examinations showed no elevation of tumor markers. He underwent left and caudate lobectomy of the liver. The diagnosis of intrahepatic cholangiocarcinoma with sarcomatous stroma was based on thorough pathologic examination and immunohistochemical staining. The tumor exhibited adenocarcinomatous and sarcomatous components; the adenocarcinomatous element was positive for epithelial markers, the sarcomatous element was positive for mesenchymal markers, but negative for epithelial markers. The patient made an uneventful recovery after surgery. At present, 14 months after surgery, he remains well with no evidence of tumor recurrence.
\end{abstract}

Conclusions: We report an unusual case of hepatic carcinosarcoma (intrahepatic cholangiocarcinoma with sarcomatous stroma) and discuss the etiology and prognosis of this rare disease.

Keywords: Hepatic carcinosarcoma, Intrahepatic cholangiocarcinoma, Etiology, Radiology and pathology

\section{Introduction}

Hepatic carcinosarcoma (HCS) is a rare tumor, which has been defined by the World Health Organization (WHO) as a malignant tumor containing an intimate mixture of carcinomatous (either hepatocellular carcinoma [HCC] or intrahepatic cholangiocarcinoma [ICC]) and sarcomatous elements [1]. The incidence of primary hepatic sarcoma is very low, but sarcomatous change often occurs in several epithelial tumors (including HCC) [2, 3]. Although carcinosarcoma with HCC has occasionally been reported [3-7], few reports of ICC with carcinosarcoma have been reported in English. Because of the scarcity of

\footnotetext{
*Correspondence: yugawa_k@surg2.med.kyushu-u.ac.jp

${ }^{1}$ Department of Surgery and Science, Graduate School of Medical Sciences, Kyushu University, Maidashi 3-1-1, Higashi-ku, Fukuoka 812-8582, Japan ${ }^{2}$ Department of Anatomic Pathology, Graduate School of Medical Sciences, Kyushu University, Maidashi 3-1-1, Higashi-ku, Fukuoka 812-8582, Japan Full list of author information is available at the end of the article
}

these reports, preoperative diagnosis of ICC with carcinosarcoma is challenging; little is known about its etiology and prognosis.

We herein present a very rare case of primary intrahepatic cholangiocarcinoma with sarcomatous stroma, confirmed by pathology following resection, and discuss the etiology and prognosis of its radiological imaging and pathology.

\section{Case presentation}

A 60-year-old man was admitted to our hospital with a liver tumor, which was discovered during fluorodeoxyglucose positron emission tomography-computed tomography (PET-CT) as a cancer examination. He had a history of hepatitis B virus infection (positive for hepatitis $B$ virus antigen), but was asymptomatic, showed no 
positive signs when examined, and had not had any medical interventions.

Analysis of serum tumor markers showed no elevated carbohydrase antigen-19-9 $(11.2 \mathrm{U} / \mathrm{ml})$, carbohydrase antigen-125 $(18.1 \mathrm{U} / \mathrm{ml})$, or carcinoembryonic antigen $(1.0 \mathrm{ng} / \mathrm{ml})$. Other parameter levels were within normal ranges. Gastroscopy and colonoscopy also showed normal findings.

Plane computed tomography (CT) scan revealed a well-defined low-density mass, $6.0 \mathrm{~cm}$ in diameter, in the caudate liver (Fig. 1a), which showed two different components in the enhanced CT scan. Contrast-enhanced CT scan showed the right tumor enhancement during the arterial phase and delayed washout in the late phase, but showed the left component as a hypovascular lesion (Fig. 1b-d). Magnetic response imaging (MRI) showed both of these components with low intensity on T1weighted images (Fig. 2a), and right component of iso-high intensity and left component of heterogeneously high on T2-weighted images (Fig. 2b). It also showed higher intensity than with normal liver parenchyma on diffusion-weighted imaging (DWI), with a high $b$ value of 1000 (Fig. 2c). Apparent diffusion coefficient (ADC) mean values of these two separated components were $1.19 \times 10^{-3} \mathrm{~mm}^{2} / \mathrm{s}$ (right component) and $1.95 \times 10^{-3}$ $\mathrm{mm}^{2} / \mathrm{s}$ (left component). It was described as a high-intensity mass on the ADC map (Fig. 2d). Gadolinium-ethoxybenzyl-diethylene-triaminepentaaceticacid (Gd-EOB-DTPA)-MRI showed the right tumor as a hyperintense in the arterial phase (Fig. 2e) and the whole tumor as a hypointense mass in the hepatobiliary phase (Fig. 2f). $\left[{ }^{18} \mathrm{~F}\right]$-fluorodeoxyglucose positron tomography (FDG-PET) showed accumulation of $\left[{ }^{18} \mathrm{~F}\right]-\mathrm{FDG}$ at both components (Fig. 2g).

The preoperative diagnosis, based on the imaging studies, was an atypical ICC. After the patient underwent left and caudate lobectomy of the liver, macropathology of the resected specimen showed that the tumor measured $7.5 \mathrm{~cm}$ in the largest dimension. The cut surface showed two different components, with a well-demarcated, yellowish, and nodular lobulated solid formation in the right, and an elastic soft and cystic formation on the left (Fig. 3a).

Micropathologically, the right tumor component (indicated as a hypervascular lesion on enhanced CT) showed an adenocarcinomatous element, composed of moderately to poorly differentiated adenocarcinoma, arranged in trabecular and irregular tubular patterns, infiltrated into the liver parenchyma (Fig. 3b). The left component (which appeared with heterogeneous high intensity on T2WI) was a sarcomatous element, mainly composed of oval- to spindle-shaped cells with a focal dilated gland ductal structure (Figs. 3c and 4a). These two components were mostly separate but with a small intermingled area with well-differentiated adenocarcinomatous and sarcomatous elements. There was no evidence of transitional feature between adenocarcinomatous and sarcomatous elements. The surrounding parenchyma showed no cirrhotic change.
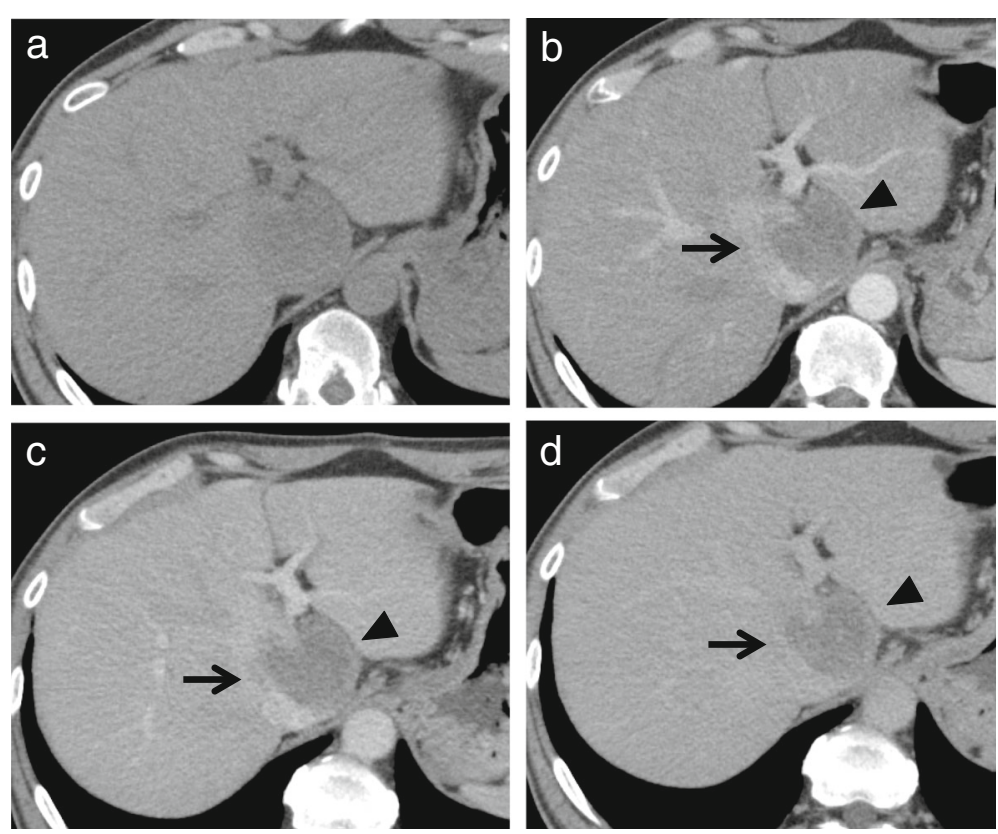

Fig. 1 Contrast-enhanced abdominal computed tomography (CT). Plane CT scan shows a well-defined low-density mass $(6.0 \mathrm{~cm}$ in diameter) in the caudate liver (a) Contrast-enhanced CT scan showed right component (arrow) of the tumor enhancement during the arterial phases (b) and delayed washout in the latter phases (c, d), but left component (arrowhead) as hypovascular lesion (b-d) 

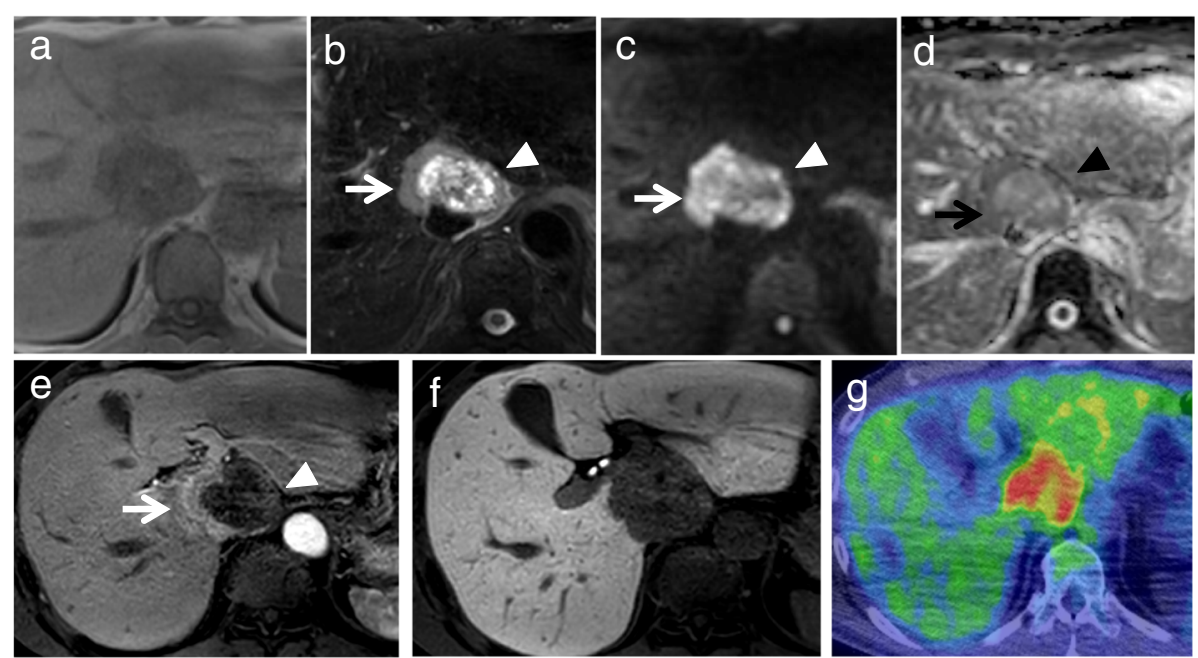

Fig. 2 Magnetic response imaging (MRI) and $\left[{ }^{18} \mathrm{~F}\right]$-fluorodeoxyglucose position tomography (FDG-PET). MR images show both components with low intensity on T1-weighted images (a) and right component (arrow) of iso-high intensity and left component (arrowhead) of heterogeneously high on T2-weighted images (b). DWl showed higher intensity than normal liver parenchyma with a high $b$ value of 1000 (c). Its ADC value was $1.19 \times 10^{-3} \mathrm{~mm}^{2} / \mathrm{s}$ (arrow on right) and $1.95 \times 10^{-3} \mathrm{~mm}^{2} / \mathrm{s}$ (arrowhead on left) (d). EOB-MRI showed right component (arrow) of the tumor as a hyperintense lesion but left component (arrowhead) as a hypointense lesion during the arterial phases (e) and hypointense mass in the hepatobiliary phase (f). FDG-PET shows accumulation of $\left[{ }^{18} \mathrm{~F}\right]$-FDG at both components (g)

In immunohistochemical (IHC) tests, the adenocarcinoma cells were positive for cytokeratin-7 (CK7), cytokeratin-19 (CK19), CD56, and epithelial membrane antigen (EMA), but negative for hepatocellular carcinoma markers such as Glypican-3 (date not shown). There were no histologic elements suggesting HCC. The sarcomatous cells were positive for S-100, $\alpha$-smooth muscle actin (SMA), and CD10, but negative for CK7, CK19, CD56, and EMA (Fig. 4b-g). The Ki67 index was $22 \%$ in the sarcomatous elements (Fig. 4h). These findings led to a pathological diagnosis of carcinosarcoma (ICC with sarcomatous stroma). The patient recovered uneventfully from the surgery, and at present, 14 months later, he remains well with no evidence of tumor recurrence.

\section{Conclusions}

Primary HCS is very rare worldwide, comprising only $1.8 \%$ to $9.4 \%$ of surgical or autopsy HCC cases $[3,8]$. Few cases have been reported in the English language journals and most have been of HCS in HCCs. However,
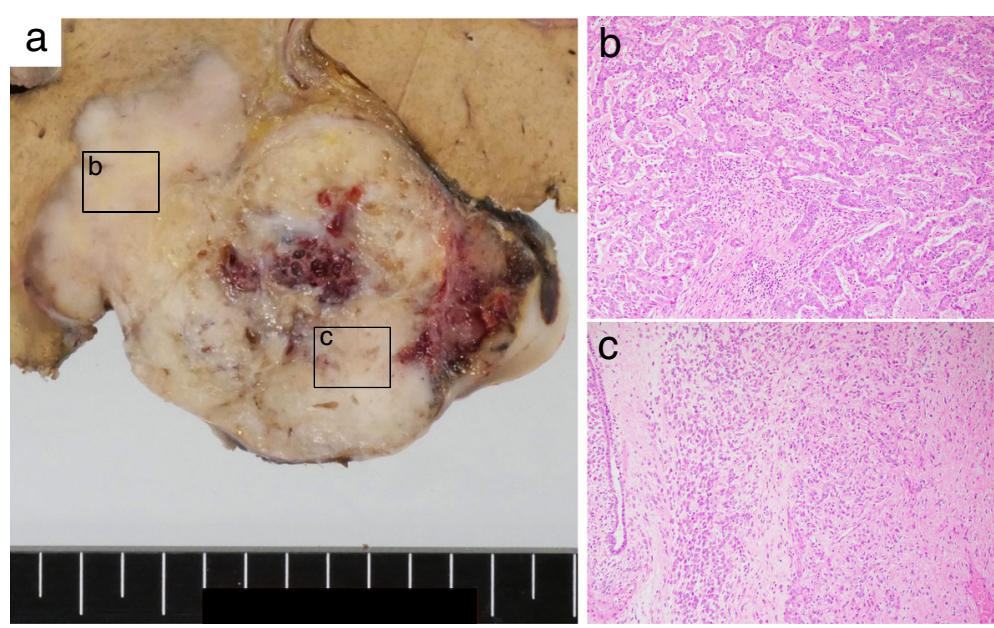

Fig. 3 Macroscopic and microscopic findings of sarcomatous ICC. a Cut surface shows (right) a well-demarcated, yellowish, nodular lobulated solid component and (left) an elastic soft, cystic component. Micropathologically, b the right component was a moderately-to-poorly differentiated adenocarcinoma, with a trabecular and irregular tubular pattern, infiltrated into liver parenchyma (hematoxylin and eosin [HE] staining $\times 100$ ). $\mathbf{c}$ The left component was sarcomatous, mainly composed of oval- to spindle-shaped cells with focal dilated gland ductal structure $(H \& E \times 100)$ 

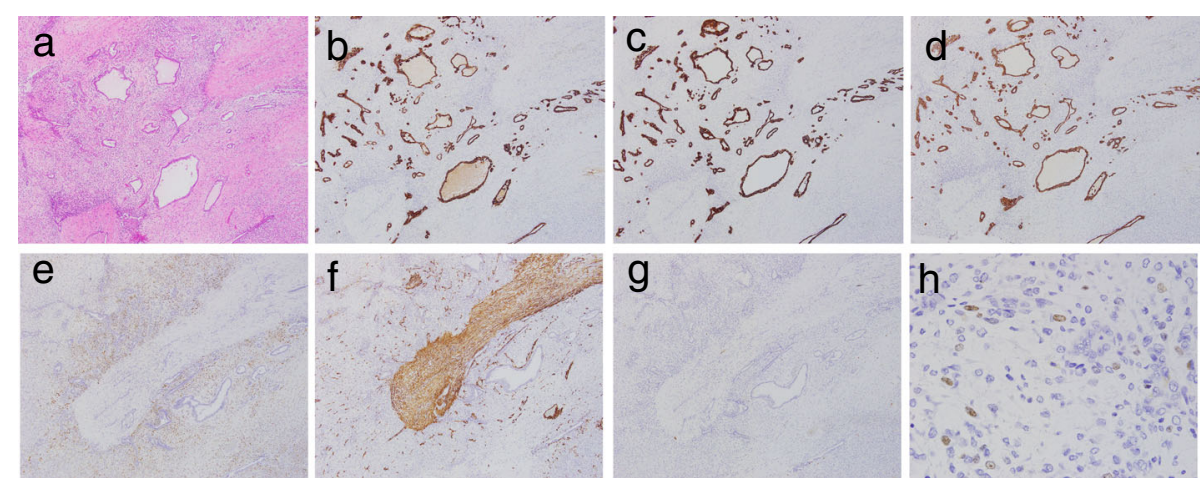

Fig. 4 Immunohistochemical staining. Microscopic findings for carcinomatous and sarcomatoid mixed area. a H\&E staining revealed that adenocarcinoma cells were positive for $\mathbf{b}$ CK7, c CK19, and $\mathbf{d}$ EMA; and sarcomatous cells were positive for e S-100, f SMA, and $\mathbf{g}$ CD10 (a-g: $\times 100)$, but negative for CK7, CK19 and EMA. $\mathbf{h}$ Ki67 index was 22\% in the sarcomatous elements ( $\times 400)$

cases of primary HCS in ICCs are even more uncommon and ICC with carcinosarcoma has a much worse prognosis than simple ICC [9].

In 1989, Craig et al. [10] first reported liver carcinosarcomas as hepatic tumors with both an HCC and a non-spindle cell sarcoma and excluded non-hepatocytic epithelial elements. According to the WHO definition, $\mathrm{HCS}$ is "a malignant tumor containing an intimate mixture of carcinomatous (either hepatocellular or cholangiocarcinoma) and sarcomatous elements." Both the WHO and Craig et al. distinguished HCS from collision tumors, and from carcinomas with foci of spindle-shaped epithelial cells, and included tumors designated as "hepatoblastoma, malignant mixed tumor, spindle cell carcinoma, or sarcomatoid carcinoma" [1]. Still, how to distinguish carcinosarcoma from sarcomatoid carcinoma is controversial. Rosai [11] suggested that such mixed tumors should be diagnosed as spindle cell carcinoma or sarcomatoid carcinoma when the sarcomatous component is predominantly composed of spindle cells, but the epithelial cells are still morphologically and immunohistochemically identifiable. Wang et al. [12] suggested the absence of significant differences in survival rates and morphologies of sarcomatous components between sarcomatoid carcinoma and carcinosarcoma, which implies that distinguishing between primary sarcomatoid carcinoma and carcinosarcoma of the liver is clinically unnecessary [12]. Based on our pathological and IHC studies, and according to the definitions of WHO and Rosai, we diagnosed this tumor as "hepatic carcinosarcoma."

We searched PubMed to identify the published case reports of ICC with sarcomatous change in the English literature and used the terms "liver," "sarcomatous," "sarcomatoid," "carcinosarcoma," and "cholangiocarcinoma." We reviewed the identified 27 patients, including our patient, the characteristics of which we here summarize (Table 1) [13-29]. In radiological images, low-density mass with enhancement by contrast medium on $\mathrm{CT}$, hypointensity on T1WI, and hyperintensity on T2WI are reported to be key sarcomatous ICC features [30]. As shown in Table 1, the identified radiological characteristics are similar to those of sarcomatous ICC. As in the previous reports, the radiological images of the distinguished sarcomatous component in the present case might be identical with the dominant sarcomatoid ICC. However, the adenocarcinoma component might differ from the ordinary ICC; hypervascular ICC is considered to have less malignant potential than other ICCs [31]. Nevertheless, the ADC mean value of two different components was $1.19 \times 10^{-3} \mathrm{~mm}^{2} / \mathrm{s}$ (ICC component) and $1.95 \times 10^{-3} \mathrm{~mm}^{2} / \mathrm{s}$ (sarcomatous component), respectively. Lower mean ADC value is associated with more aggressive histopathology and poorly differentiated ICCs [32]. The ADC value indicates that the ICC component has more malignant potential than the sarcomatous component.

In previous reports, pathologists have proposed two pathogeneses for HCS. One theory, supported by IHC, holds that HCSs develop from multipotent progenitor or stem cells of the liver. This theory indicates dual differentiation by an immature malignant cell and shows that combination tumors may originate from single totipotent stem cell, which differentiates in separate epithelial and mesenchymal directions [33]. The alternative theory, which is based on observation of transitional zones, posits that conventional tumor cells transform or dedifferentiate into sarcomatous components from hepatocellular or cholangiocellular carcinoma. Some reports support the idea that malignant cells might change into multipotent immature cells [34, 35].

In our case, based on IHC findings for our carcinosarcoma specimen, we support the theory that the carcinosarcoma developed from hepatic progenitor cells or stem cells, which differentiates separately into both epithelial and mesenchymal elements. These two different elements were largely separated, although a focal area 


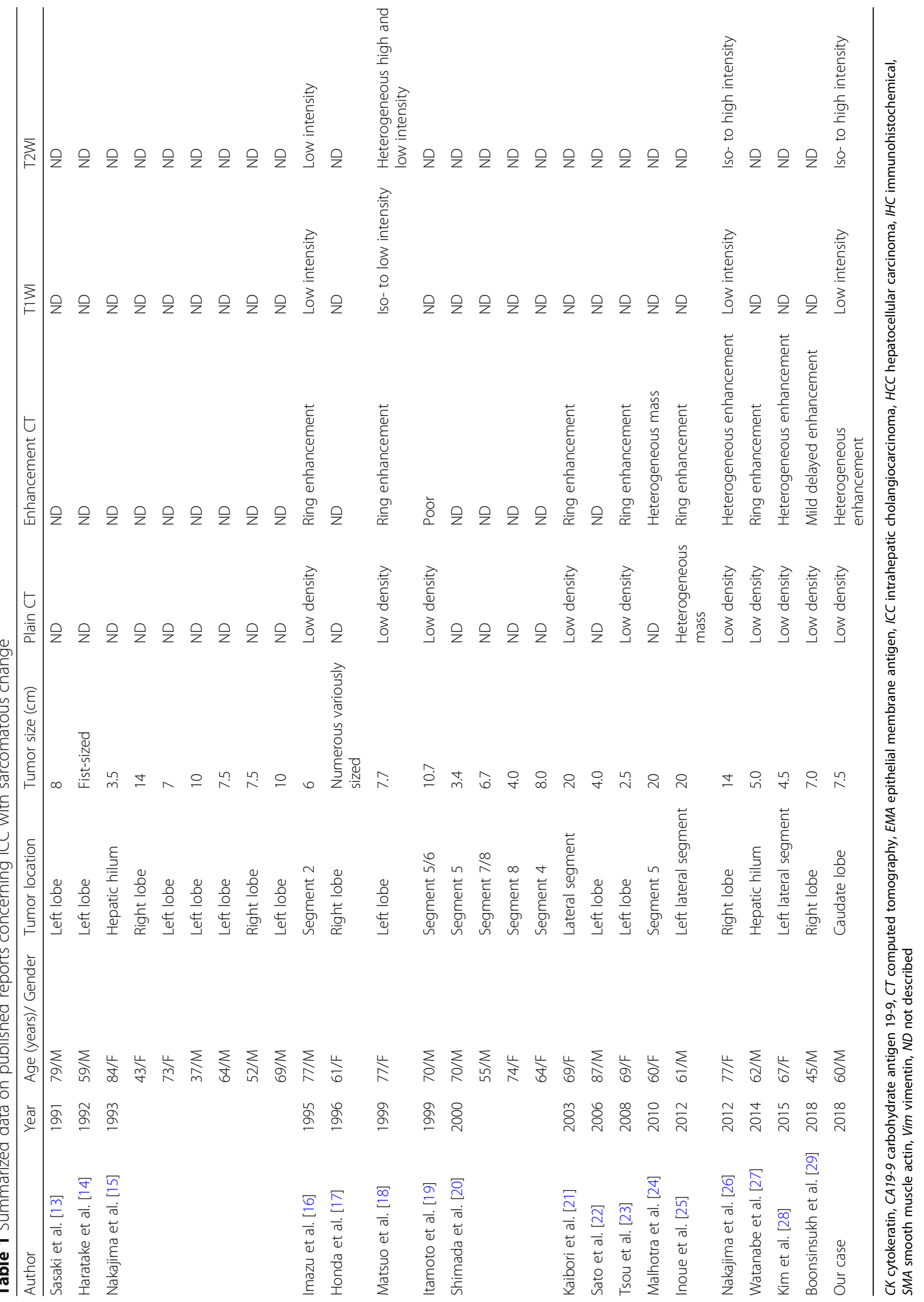




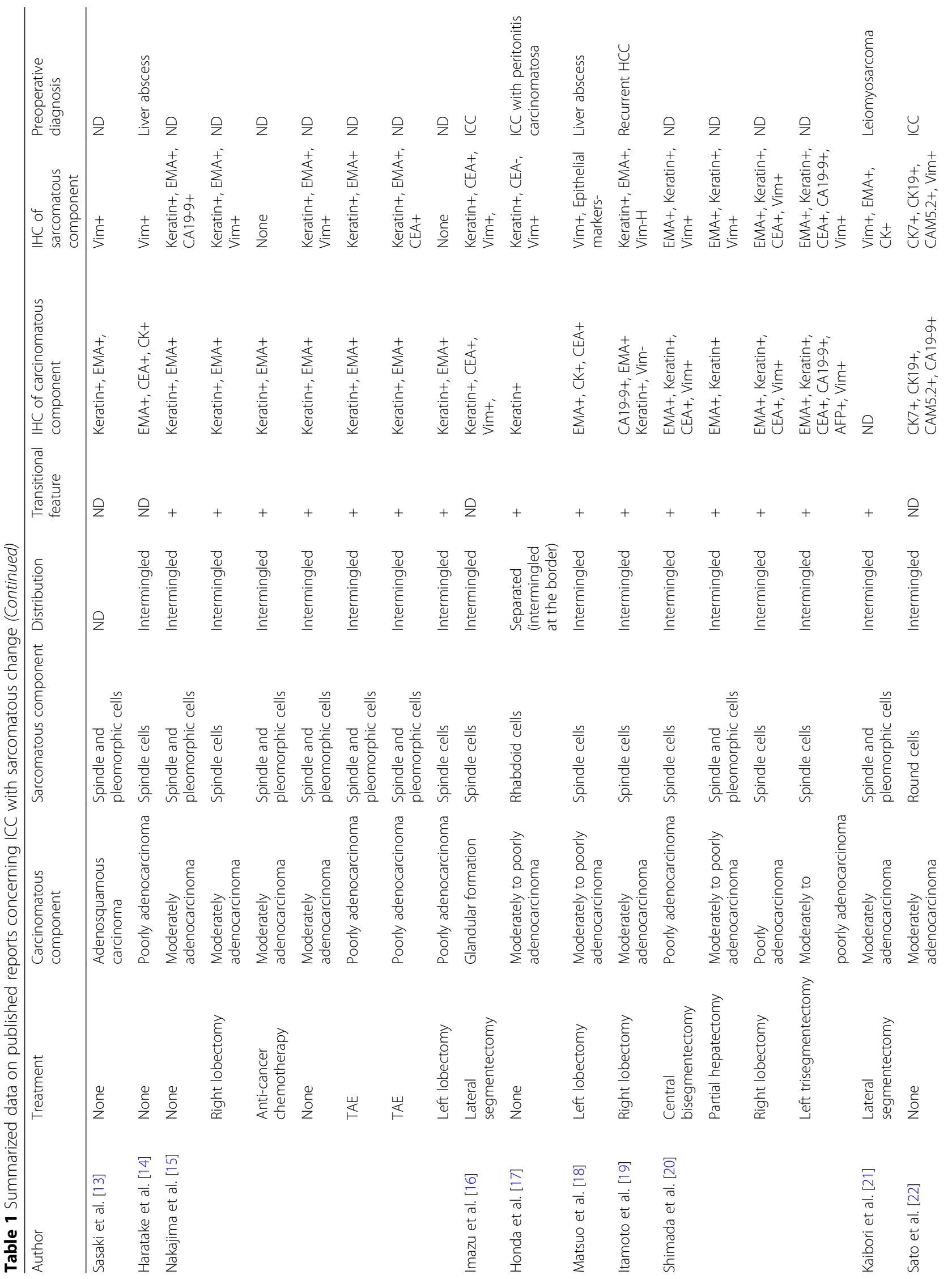




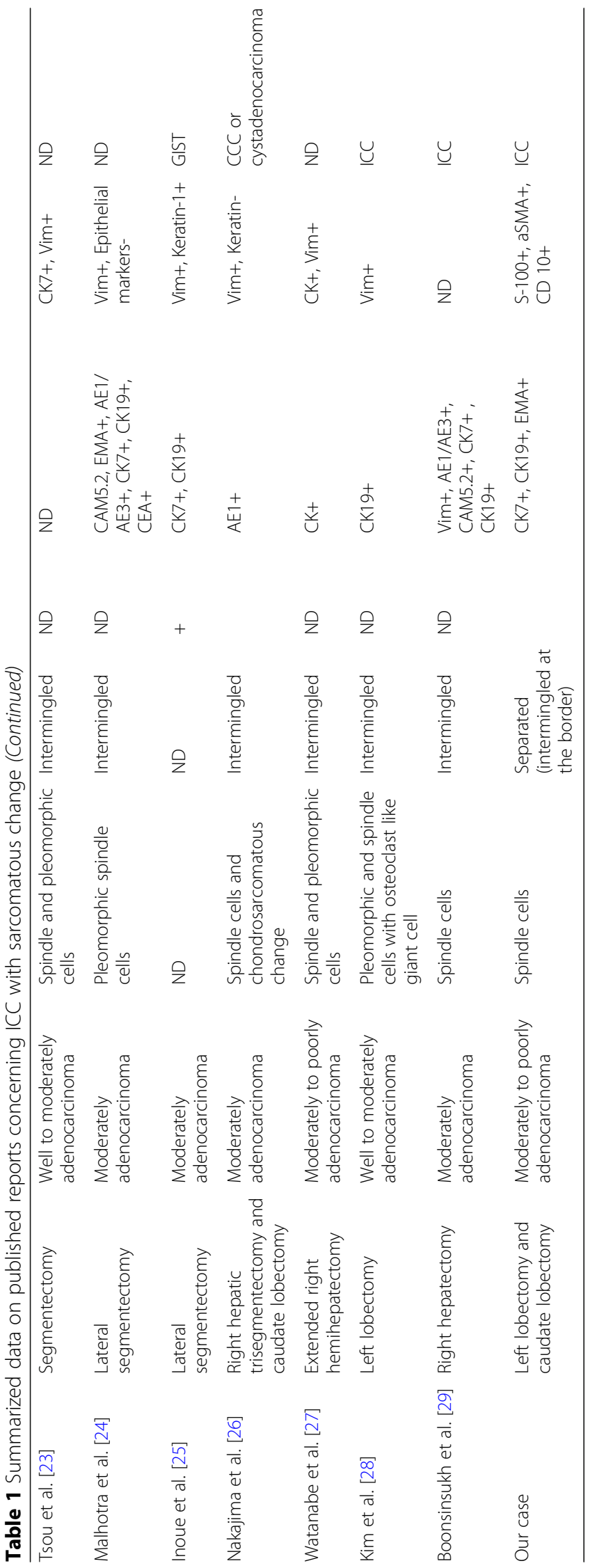


was intermingled, with small amounts of adenocarcinomatous elements and sarcomatous elements, with no evidence of transitional zones. As shown in Table 1, distribution of these two elements was intermingled and transitional area was observed in the most cases; however, our histological results were different patterns from previous reported cases. Moreover, only the adenocarcinoma cells were invading the hepatic parenchyma, whereas the sarcomatous element proliferated in the caudate without invading, except for the intra-inferior vena cava. In these morphological features, adenocarcinomatous and sarcomatous elements can have different properties. Interestingly, our IHC results revealed that the adenocarcinoma elements were positive for epithelial markers (CK7, CK19, CD56, and EMA) but negative for mesenchymal markers (S-100, alpha-SMA, and CD10), whereas the sarcomatous elements were positive for mesenchymal markers, but negative for epithelial markers. To our knowledge, no previous cases of separated ICC carcinomatous and sarcomatous components shown by radiological and IHC findings have been reported.

In conclusion, we reported an unusual case of hepatic carcinosarcoma (ICC with sarcomatous stroma). The results of the present case report supported the etiological theory that sarcomatous elements developed from progenitor or stem cells, rather than redifferentiated from epithelial elements. More epidemiological and pathological data will be further required to confirm the etiology and prognosis of the rare malignant tumor.

\section{Abbreviations \\ ADC: Apparent diffusion coefficient; CK19: Cytokeratin 19; CK7: Cytokeratin 7; CT: Computed tomography; DWI: Diffuse weighted imaging; EMA: Epithelial membrane antigen; EOB-MRI: Gadolinium ethoxybenzyl diethylenetriamine pentaacetic acid-enhanced magnetic response imaging; FDG-PET: $\left[{ }^{18} \mathrm{~F}\right]-$ fluorodeoxyglucose position tomography; HCC: Hepatocellular carcinoma; HCS: Hepatic carcinosarcoma; ICC: Intrahepatic cholangiocarcinoma; IHC: Immunohistochemical; MRI: Magnetic response imaging; PET- CT: Fluorodeoxyglucose positron emission tomography-computed tomography; SMA: Smooth muscle actin; WHO: World Health Organization.}

\section{Acknowledgements}

We thank Marla Brunker, from Edanz Group (www.edanzediting.com/ac) for editing a draft of this manuscript.

\section{Funding}

Not applicable.

\section{Availability of data and materials}

Not applicable.

\section{Authors' contributions}

$K Y$ acquired the data and drafted the manuscript. KY, YM, TT, and TY performed the surgeries. All other authors attended the patient postoperatively. All authors read and approved the final manuscript.

\section{Ethics approval and consent to participate}

No applicable.

\section{Consent for publication}

Oral informed consent was obtained from the patient for the publication of this case report and accompanying images.

\section{Competing interests}

The authors declare that they have no competing interests.

\section{Publisher's Note}

Springer Nature remains neutral with regard to jurisdictional claims in published maps and institutional affiliations.

\section{Author details}

${ }^{1}$ Department of Surgery and Science, Graduate School of Medical Sciences, Kyushu University, Maidashi 3-1-1, Higashi-ku, Fukuoka 812-8582, Japan. ${ }^{2}$ Department of Anatomic Pathology, Graduate School of Medical Sciences, Kyushu University, Maidashi 3-1-1, Higashi-ku, Fukuoka 812-8582, Japan. ${ }^{3}$ Department of Clinical Radiology, Graduate School of Medical Sciences, Kyushu University, Maidashi 3-1-1, Higashi-ku, Fukuoka 812-8582, Japan. ${ }^{4}$ Department of Pathology and Microbiology, Faculty of Medicine, Saga University Hospital, Saga, Japan.

Received: 19 October 2018 Accepted: 12 November 2018 Published online: 26 November 2018

\section{References}

1. Ishak KG, Anthony PP, Niederau C, et al. Mesenchymal tumours of the liver. In: Stanley RH, Lauri AA, editors. WHO international histological classification of tumours pathology \& genetics of tumours of the digestive system. Lyon: IARC Press; 2000. p. p198.

2. Matsuoka T, Watanabe H, Enjoji M. Pseudosarcoma and carcinosarcoma of esophagus. Cancer. 1976;37:1546-55.

3. Kakizoe S, Kojiro M, Nakashima T. Hepatocellular carcinoma with sarcomatous change: clinicopathologic and immunohistochemical studies of 14 cases. Cancer. 1987;59:310-26.

4. Akasofu M, Kawahara E, Kaji K, Nakanishi I. Sarcomatoid hepatocellular carcinoma showing rhabdomyoblastic differentiation in the adult cirrhotic liver. Virchows Arch. 1999;434:511-5.

5. Fu Y, Kobayashi S, Kushida Y, et al. Sarcomatoid hepatocellular carcinoma with chondroid variant: case report with immunohistochemical findings. Pathol Int. 2000;50:919-22.

6. Koda M, Maeda Y, Matsunaga Y, Mimura K, Murawaki Y, Horie Y. Hepatocellular carcinoma with sarcomatous change arising after radiofrequency ablation for well-differentiated hepatocellular carcinoma. Hepatol Res. 2003;27:163-7.

7. Yoshida N, Midorikawa Y, Kajiwara T, et al. Hepatocellular carcinoma with sarcomatoid change without anticancer therapies. Case Rep Gastroenterol. 2013;7:169-74.

8. Maeda T, Adachi E, Kajiyama K, Takenaka K, Sugimachi K, Tsuneyoshi M. Spindle cell hepatocellular carcinoma. A clinicopathologic and immunohistochemical analysis of 15 cases. Cancer. 1996;77:51-7.

9. Okabayashi T, Shima Y, Iwata J, Morita M. Surgical outcomes for 131 cases of carcinosarcoma of the hepatobiliary tract. J Gastroenterol. 2014;49:982-91.

10. Craig JR, Peters RL, Edmondson HA. Tumors of the liver and intrahepatic bile duct. In: Hartmann WH, Sobin LH, editors. Atlas of tumor pathology, second series, fascile 26. Washington: Armed Forces Institute of Pathology; 1988. p. 179-80.

11. Rosai J. Rosai and Ackerman's surgical pathology. Edinburgh: Mosby; 2004

12. Wang QB, Cui BK, Weng JM, Wu QL, Lin XJ. Clinicopathological characteristics and outcome of primary sarcomatoid carcinoma and carcinosarcoma of the liver. J Gastrointest Surg. 2012;16:1715-26.

13. Sasaki M, Nakanuma $Y$, Nagai $Y$, et al. Intrahepatic cholangiocarcinoma with sarcomatous transformation: an autopsy case. J Clin Gastroenterol. 1991;13: 220-5.

14. Haratake J, Yamada $\mathrm{H}$, Horie A, et al. Giant cell tumor-like cholangiocarcinoma associated with systemic cholelithiasis. Cancer. 1992;69: 2444-8.

15. Nakajima T, Tajima Y, Sugano I, et al. Intrahepatic cholangiocarcinoma with sarcomatous change. Clinicopathologic and immunohistochemical evaluation of seven cases. Cancer. 1993;72:1872-7.

16. Imazu H, Ochiai M, Funabiki T. Intrahepatic sarcomatous cholangiocarcinoma. J Gastroenterol. 1995;30:677-82.

17. Honda M, Enjoji M, Sakai H, et al. Case report: intrahepatic cholangiocarcinoma with rhabdoid transformation. J Gastroenterol Hepatol. 1996;11:771-4. 
18. Matsuo S, Shinozaki T, Kanematsu T, et al. Intrahepatic cholangiocarcinoma with extensive sarcomatous change: report of a case. Jpn J Surg. 1999;29: 560-3.

19. Itamoto T, Asahara T, Katayama K, et al. Double cancer - hepatocellular carcinoma and intrahepatic cholangiocarcinoma with a spindle-cell variant. J Hepato-Biliary-Pancreat Surg. 1999;6:422-6.

20. Shimada M, Takenaka K, Rikimaru T, et al. Characteristics of sarcomatous cholangiocarcinoma of the liver. Hepatogastroenterology. 2000;47:956-61.

21. Kaibori M, Kawaguchi Y, Yokoigawa N, et al. Intrahepatic sarcomatoid cholangiocarcinoma. J Gastroenterol. 2003;38:1097-101.

22. Sato K, Murai H, Ueda $Y$, et al. Intrahepatic sarcomatoid cholangiocarcinoma of round cell variant: a case report and immunohistochemical studies. Virchows Arch. 2006;449:585-90.

23. Tsou YK, Wu RC, Hung CF, et al. Intrahepatic sarcomatoid cholangiocarcinoma: clinical analysis of seven cases during a 15-year period. Chang Gung Med J. 2008;31:599-605.

24. Malhotra S, Wood J, Mansy T, et al. Intrahepatic sarcomatoid cholangiocarcinoma. J Oncol. 2010;2010:701476. https://doi.org/10.1155/ 2010/701476.

25. Inoue Y, Lefor AT, Yasuda Y. Intrahepatic cholangiocarcinoma with sarcomatous changes. Case Rep Gastroenterol. 2012;6:1-4.

26. Nakajima T, Okumura A. A case of huge intrahepatic cholangiocarcinoma. J Jpn Soc Gastroenterol. 2012;109:1590-7.

27. Watanabe $G$, Uchinami $H$, Yamamoto $Y$, et al. Prognosis analysis of sarcomatous intrahepatic cholangiocarcinoma from a review of the literature. Int J Clin Oncol. 2014;19:490-6.

28. Kim HM, Kim H, Park YN. Sarcomatoid cholangiocarcinoma with osteoclastlike giant cells associated with hepatolithiasis: a case report. Clin Mol Hepatol. 2015;21(3):309-13.

29. Boonsinsukh $T$, Viriyaroj $V$, Trongwongsa $T$, et al. Intrahepatic sarcomatous cholangiocarcinoma: case report and review of the literature. Case Rep Surg. 2018;2018:3862575. https://doi.org/10.1155/2018/3862575.

30. Gu KW, Kim YK, Min JH, Ha SY, Jeong WK. Imaging features of hepatic sarcomatous carcinoma on computed tomography and gadoxetic acidenhanced magnetic resonance imaging. Abdom Radiol. 2017;42:1424-33.

31. Fujita N, Asayama Y, Nlshie A, Honda H. Mass-forming intrahepatic cholangiocarcinoma: enhancement patterns in the arterial phase of dynamic hepatic CT - correlation with clinicopathological findings. Eur Radiol. 2016;27:498-506

32. Lewis S, Besa C, Wagner M, Taouli B. Prediction of histopathologic findings of intrahepatic cholangiocarcinoma: qualitative and quantitative assessment of diffusion-weighted imaging. Eur Radiol. 2018;28:2047-57.

33. Fayyazi A, Nolte W, Oestmann JW, Sattler B, Ramadori G, Radzun HJ. Carcinosarcoma of the liver. Histopathology. 1998;32:385-7.

34. Kubosawa H, Matsuzaki O, Kondo Y, Takao M, Sato N. Carcinosarcoma of the prostate. Acta Pathol Jpn. 1993:43:209-14.

35. Lin YS, Wang TY, Lin JC, Chen MJ, et al. Hepatic carcinosarcoma: clinicopathologic features and a review of the literature. Ann Hepatology. 2013;12:495-500

\section{Submit your manuscript to a SpringerOpen ${ }^{\oplus}$ journal and benefit from:}

- Convenient online submission

- Rigorous peer review

- Open access: articles freely available online

- High visibility within the field

- Retaining the copyright to your article

Submit your next manuscript at $>$ springeropen.com 